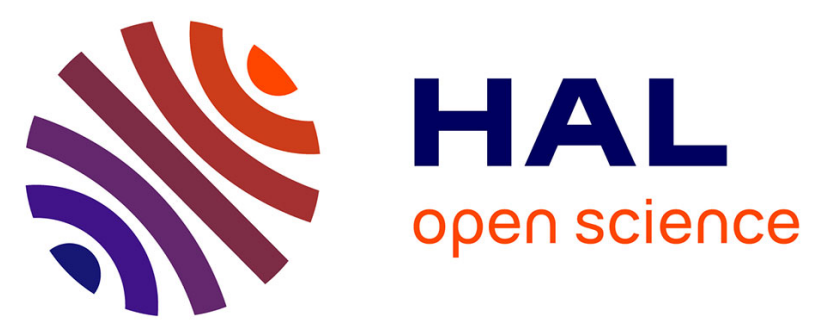

\title{
Interfacial Dzyaloshinskii-Moriya Interaction, Perpendicular Magnetic Anisotropy and damping in $\mathrm{CoFeB} /$ oxide-based systems
}

I. Benguettat-El Mokhtari, Y. Roussigne, S. M Cherif, S. Auffret, C. Baraduc, Hélène Béa, M. Belmeguenai

\section{To cite this version:}

I. Benguettat-El Mokhtari, Y. Roussigne, S. M Cherif, S. Auffret, C. Baraduc, et al.. Interfacial Dzyaloshinskii-Moriya Interaction, Perpendicular Magnetic Anisotropy and damping in CoFeB/oxidebased systems. IEEE Transactions on Magnetics, In press, pp.1-1. 10.1109/TMAG.2021.3088995 . hal-03268994

\section{HAL Id: hal-03268994 \\ https://hal.science/hal-03268994}

Submitted on 23 Jun 2021

HAL is a multi-disciplinary open access archive for the deposit and dissemination of scientific research documents, whether they are published or not. The documents may come from teaching and research institutions in France or abroad, or from public or private research centers.
L'archive ouverte pluridisciplinaire HAL, est destinée au dépôt et à la diffusion de documents scientifiques de niveau recherche, publiés ou non, émanant des établissements d'enseignement et de recherche français ou étrangers, des laboratoires publics ou privés. 


\title{
Interfacial Dzyaloshinskii-Moriya Interaction, Perpendicular Magnetic Anisotropy and damping in $\mathrm{CoFeB} / \mathrm{oxide}-\mathrm{based}$ systems
}

\author{
I. Benguettat-El Mokhtari ${ }^{1,2}$, Y. Roussigné ${ }^{1}$, S. M. Chérif ${ }^{1}$, S. Auffret ${ }^{3}$, C. Baraduc ${ }^{3}$, H. Béa ${ }^{3}$ and M. Belmeguenai ${ }^{1}$ \\ ${ }^{1}$ Université Sorbonne Paris Nord, LSPM, CNRS, UPR 3407, F-93430 Villetaneuse, France \\ ${ }^{2}$ LPCMME, Université Oran1, BP1524, El M'naouar 31100 Oran, Algérie \\ ${ }^{3}$ Université Grenoble Alpes, CEA, CNRS, Grenoble INP, IRIG, SPINTEC, F-38000 Grenoble, France
}

\begin{abstract}
Perpendicular magnetic anisotropy (PMA), spin pumping induced damping and interfacial Dzyaloshinskii-Moriya interaction (iDMI), which are spin-orbit coupling-related phenomena of utmost importance for applications, were experimentally investigated in as grown and $225^{\circ} \mathrm{C}$ annealed $\mathrm{CoFeB} / \mathrm{PtO}_{\mathrm{x}}, \mathrm{CoFeB} / \mathrm{TaO}_{\mathrm{x}}$ and $\mathrm{Ta} / \mathrm{CoFeB} / \mathrm{TaO}_{\mathrm{x}}$ systems by means of vibrating sample magnetometry, microstrip ferromagnetic resonance and Brillouin light scattering techniques. By varying $\mathrm{Cos}_{8} \mathrm{Fe}_{72} \mathrm{~B}_{20}(\mathrm{CoFeB})$ thickness in the range 0.8-10 nm, the effect of Ta buffer layer on anisotropy and damping was first studied, where a large surface magnetic anisotropy $\left(\mathrm{K}_{\mathrm{s}}=\mathbf{2 . 1} \pm \mathbf{0 . 1 6} \mathrm{erg} / \mathrm{cm}^{2}\right)$ was measured in the unbuffered $\mathrm{CoFeB} / \mathrm{TaOx}(0.8 \mathrm{~nm})$ system most likely due to their higher roughness induced by the substrate. $\mathrm{K}_{\mathrm{s}}$ degrades significantly for CoFeB film thickness below $2 \mathrm{~nm}$ where spontaneous perpendicular magnetization was found to be impossible without Ta buffer layer. PMA, iDMI and damping of as-deposited and $225^{\circ} \mathrm{C}$ annealed $\mathrm{CoFeB}(1.5 \mathrm{~nm}) / \mathrm{PtO}_{\mathrm{x}}$ systems were measured as a function of $\mathrm{PtO}_{\mathrm{x}}$ thickness in the range 0.7-1.6 nm. Their strong dependence versus the $\mathrm{PtO}_{\mathrm{x}}$ thickness was attributed to the decrease of the magnetic dead layer as $\mathbf{P t O}_{\mathrm{x}}$ thickness increases. Linear dependence of damping versus PMA constant were obtained confirming their relation with the spin orbit coupling. Moreover, annealing increases PMA and the effective mixing conductance probably due to the enhancement of the CoFeB crystal structure and interfaces.
\end{abstract}

Index Terms - Ferromagnetic resonance, interfacial Dzyaloshinskii-Moriya interaction, magnetic damping, magnetic anisotropy,

\section{INTRODUCTION}

$\mathrm{S}$ INCE THE DISCOVERY of the giant magnetoresistance effect $[1,2]$, extensive efforts have been devoted to the development of spintronics, where devices use in addition to the charge of the electron its quantum property spin, unlike conventional electronic semiconductors. Smaller, faster and efficient, these words describe the features of spintronics devices for data storage [3], including nonvolatility, high speed and low power consumption. Magnetic tunnel junction (MTJ) [4], and the skyrmions racetrack memory [5, 6], remain the most promising spintronic devices. However, it was found that realizing such high-performance spintronic devices, is quite dependent on multilayers based systems where heavy-metal (HM)/ferromagnetic (FM) interface play a crucial role. Indeed, spin-orbit coupling (SOC)-related phenomena at HM/FM interface, such as perpendicular magnetic anisotropy (PMA) [7], spin pumping [8, 9], and interfacial Dzyaloshinskii-Moriya interaction (iDMI) $[10,11]$, are the most interesting.

PMA materials are one of the best candidates for magnetic storage. Indeed, it enhances thermal stability and induced low driving current in Magnetic random access memory (MRAM) [12], where MTJ is the unit cell. PMA was found to exist at $\mathrm{HM} / \mathrm{FM}[7]$ and also in FM/Metal Oxide $\left(\mathrm{MO}_{\mathrm{x}}\right)$ interface [13, 14]. Among PMA materials, $\mathrm{CoFeB} / \mathrm{MgO}$-based structures are widely studied, due to their high tunnel magnetoresistance [15,

Manuscript received April 1, 2015; revised May 15, 2015 and June 1, 2015; accepted July 1, 2015. Date of publication July 10, 2015; date of current version July 31, 2015. (Dates will be inserted by IEEE; "published" is the date

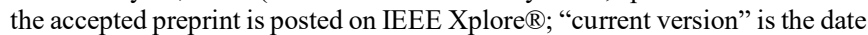
the typeset version is posted on Xplore $\left.{ }^{\circledR}\right)$. Corresponding author: $M$. Belmeguenai (e-mail: belmeguenai.mohamed@univ-paris13.fr). IEEE TRANSACTIONS ON MAGNETICS discourages courtesy authorship;

Color versions of one or more of the figures in this paper are available online at http://ieeexplore.ieee.org.

Digital Object Identifier (inserted by IEEE).
16] and low magnetic damping [16, 17]. However, to improve MTJ performance, thermal stability is still the major issue. Since then, plenty of endeavors have been put to find the best CoFeB-based structure showing strong PMA, by investigating various $\mathrm{HM}$ and $\mathrm{MO}_{\mathrm{x}}$ materials [14].

Besides PMA, "iDMI", another SOC effect is also widely investigated for the future spintronic devices to encode bits. Indeed, iDMI which is an antisymmetric exchange interaction, combined with PMA can give rise to a variety of chiral magnetic textures such as spin spirals $[18,19]$ and skyrmion lattices [20, 21]. A sufficiently large iDMI can also stabilize chiral domain walls [22]. Knowing that the existence of the iDMI requires the broken inversion symmetry, the large SOC at $\mathrm{HM} / \mathrm{FM}$ interface also play determining role since it favors significant iDMI following the Fert-Levy model [21]. Therefore, considerable effort has been made to search for materials with large DMI or new possibilities to enhance DMI for the skyrmion/domain wall based spintronic devices. Until recently, the iDMI investigation focused on the FM/HM interface, nevertheless it can also exist at FM/Oxide interfaces attributed to the Rashba interfacial coupling [23], which was analyzed from theoretical studies [23, 24] and lately demonstrated experimentally $[25,26]$. However, the understanding of the physical triggering mechanisms is still missing. Particularly, the effect of oxygen concentration and annealing combined with different metal oxides on the amplitude and sign of iDMI.

In this work, we investigated the $\mathrm{CoFeB}$ thickness dependence of PMA and damping in $\mathrm{CoFeB} / \mathrm{TaO}_{x}(0.8 \mathrm{~nm})$ and $\mathrm{Ta} / \mathrm{CoFeB} / \mathrm{TaO}_{\mathrm{x}}(0.8 \mathrm{~nm})$, where a large perpendicular surface anisotropy was found for $\mathrm{CoFeB} / \mathrm{TaO}_{\mathrm{x}}(0.8 \mathrm{~nm})$. We also demonstrate the possibility of tuning and controlling iDMI, PMA and spin pumping induced damping in $\operatorname{CoFeB}(1.5$ 
$\mathrm{nm}) / \mathrm{PtO}_{\mathrm{x}}$ by varying the $\mathrm{PtO}_{\mathrm{x}}$ thickness and the oxidation level in as grown and $225^{\circ} \mathrm{C}$ annealed samples. This allowed understanding $\mathrm{PtO}_{\mathrm{x}}$ effect on these three effects. For this, Brillouin light scattering (BLS), ferromagnetic resonance with microstrip line (MS-FMR) coupled to vibrating sample magnetometery (VSM) were used.

\section{SAMPLES AND EXPERIMENTAL TECHNIQUES}

$\mathrm{Co}_{8} \mathrm{Fe}_{72} \mathrm{~B}_{20} / \mathrm{MO}_{\mathrm{x}}$ bilayers, where $\mathrm{MO}_{\mathrm{x}}=\mathrm{PtO}_{\mathrm{x}}$ or $\mathrm{TaO}_{\mathrm{x}}$, were grown at room temperature directly on thermally oxidized silicon $\left(\mathrm{Si} / \mathrm{SiO}_{2}\right)$ substrates or using $3 \mathrm{~nm}$ thick Ta buffer layer by DC magnetron sputtering having high base vacuum level (typically in the range of several $10^{-8}$ mbar). $\mathrm{TaO}_{\mathrm{x}}$ and $\mathrm{PtO}_{\mathrm{x}}$ were obtained by growing the desired Ta or Pt thickness which is then oxidized in a treatment chamber. For Ta oxidation, an oxygen pressure of $150 \mathrm{mbar}$ for $10 \mathrm{~s}$ has been used, whereas for Pt oxidation, oxygen plasma with a power of $10 \mathrm{~W}$ and oxygen pressure of $3 \times 10^{-3}$ mbar was used for $85 \mathrm{~s}$. All the samples were further capped by $\mathrm{Al}(0.5 \mathrm{~nm})$ for surface protection and in order to prevent some metallic Al to remain, which could be detrimental to gate voltage application on the stack (not shown here) [27]. To investigate the FM thickness dependence of the SOC-related phenomena and for both Tabuffered and unbuffered samples, the CoFeB thickness $\left(\mathrm{t}_{\mathrm{CFB}}\right)$ has been varied in ranges $0.8-10 \mathrm{~nm}$ and $2-10 \mathrm{~nm}$ for $\mathrm{Ta} / \mathrm{CoFeB} / \mathrm{TaO}_{\mathrm{x}}$ and $\mathrm{CoFeB} / \mathrm{TaO}_{\mathrm{x}}$, respectively. $\mathrm{The}^{\mathrm{TaO}}$ is kept constant $(0.8 \mathrm{~nm})$ and samples were then annealed at $225^{\circ} \mathrm{C}$, optimized to improve PMA [28], in vacuum for 30 minutes. Note that due to the weak measured iDMI in the previously investigated $\mathrm{Ta} / \mathrm{CoFeB} / \mathrm{TaO}_{x}$, we limited our comparison between $\mathrm{CoFeB} / \mathrm{TaO}_{\mathrm{x}}$ and $\mathrm{Ta} / \mathrm{CoFeB} / \mathrm{TaO}_{\mathrm{x}}$ to evidence the effect of Ta buffer layer to PMA and damping and only samples with $\mathrm{CoFeB}$ in the range $2-10 \mathrm{~nm}$ are considered $\mathrm{CoFeB} / \mathrm{TaO}_{\mathrm{x}}$. The second category of samples considered in this study is composed of $1.5 \mathrm{~nm}$ thick $\mathrm{CoFeB}$ grown on $\mathrm{Si} / \mathrm{SiO}_{2}$ substrate without $\mathrm{Ta}(3 \mathrm{~nm})$ underlayer and capped by Pt layer with variable thickness $\left(0.7 \mathrm{~nm} \leq \mathrm{t}_{\mathrm{t}} \leq 1.6 \mathrm{~nm}\right)$. Both as grown and $225^{\circ} \mathrm{C}$ annealed samples are considered for this category. The deposited thicknesses are determined by the opening time of the shutter that covers the target, calibrated by recording the time deposition of $30 \mathrm{~nm}$ thick films, measured by $\mathrm{x}$-ray reflectivity.

The magnetic moment at saturation has been determined from the hysteresis curves obtained by VSM, under in-plane applied magnetic field. MS-FMR was used to determine the gyromagnetic ratio, the damping and the PMA for the thicker films. BLS has been used to investigate PMA and iDMI. For this, Stokes (S) and anti-Stokes (aS) spin wave (SW) frequencies have been measured as function of the in-plane applied magnetic field and the SW vector $\left(\mathrm{k}_{\mathrm{SW}}\right)$. For PMA investigation, the field dependence of the mean frequency $\left[\left(\mathrm{F}_{\mathrm{S}}+\mathrm{F}_{\mathrm{aS}}\right) / 2\right]$, at fixed spin SW vector of $4.1 \mu \mathrm{m}^{-1}$ (incidence angle of $10^{\circ}$ ) was used, whereas, the variation of the frequency mismatch between $\mathrm{S}$ and aS lines $\left(\Delta \mathrm{F}=\mathrm{F}_{\mathrm{S}}-\mathrm{F}_{\mathrm{aS}}\right)$ versus $\mathrm{k}_{\mathrm{SW}}$ allowed to characterize iDMI strength.

\section{RESULTS AND DISCUSSIONS}

\section{A. 1- Effects of CoFeB thickness and Ta buffer layer}

The Magnetization at saturation $\left(\mathrm{M}_{\mathrm{s}}\right)$, the magnetic dead layer thickness $\left(t_{d}\right)$ and the gyromagnetic ratio $(\gamma)$ are needed for the precise characterization of iDMI, PMA and spin pumping efficiency. $M_{s}$ and $t_{d}$ have been deduced from the $\mathrm{CoFeB}$ thickness dependence of the saturation magnetic moment per unit area measured by VSM, shown in figure 1 for $\mathrm{CoFeB} / \mathrm{TaO}_{\mathrm{x}}, \mathrm{Ta} / \mathrm{CoFeB} / \mathrm{TaO}_{\mathrm{x}}$ and $\mathrm{CoFeB} / \mathrm{PtO}_{\mathrm{x}}$. The data indicate that $\mathrm{M}_{\mathrm{s}}$ values are $1470 \pm 50 \mathrm{emu} / \mathrm{cm}^{3}, 1536 \pm 60$ $\mathrm{emu} / \mathrm{cm}^{3}$ [29] and $1520 \pm 50 \mathrm{emu} / \mathrm{cm}^{3}$ for $\mathrm{CoFeB} / \mathrm{TaO}_{\mathrm{x}}(0.8 \mathrm{~nm})$, $\mathrm{Ta} / \mathrm{CoFeB} / \mathrm{TaO}_{\mathrm{x}}(0.8 \mathrm{~nm})$ and $\mathrm{CoFeB} / \mathrm{PtO}_{\mathrm{x}}(1 \mathrm{~nm})$, respectively. These high values of $\mathrm{M}_{\mathrm{s}}$ are most probably due the $\mathrm{CoFeB}$ composition which is rich in $\mathrm{Fe}$ known to have high values of $\mathrm{M}_{\mathrm{s}}$. The corresponding $\mathrm{t}_{\mathrm{d}}$ are $0.71 \pm 0.16 \mathrm{~nm}, 0.7 \pm 0.1 \mathrm{~nm}$ [29] and $1.02 \pm 0.22 \mathrm{~nm}$, respectively. This magnetic dead layer is most probably attributed to intermixing of the top $\mathrm{Ta}$ or $\mathrm{Pt}$ metals and the $\mathrm{CoFeB}$ layer when these top metals are not completely oxidized and to the interpenetrating oxygen atoms in $\mathrm{CoFeB}$ during oxidation of $\mathrm{Ta}$ or Pt, owing to the similar magnetic dead layer thickness of the unbuffered and Tabuffered $\mathrm{CoFeB} / \mathrm{TaO}_{\mathrm{x}}$ samples. This is compatible with the thicker magnetic dead layer $(1 \mathrm{~nm})$ for $\mathrm{CoFeB} / \mathrm{PtO} \mathrm{x}_{\mathrm{x}}$ since $\mathrm{Pt}$ does not generally oxidize and therefore, the oxygen penetrates more into the $\mathrm{CoFeB}$ films, increasing thus the thickness of the magnetic dead layer. In the following, all parameters will be discussed as a function of this effective thickness defined as $t_{\text {eff }}=t_{C F B}-t_{d}$, unless if explicitly mentioned.

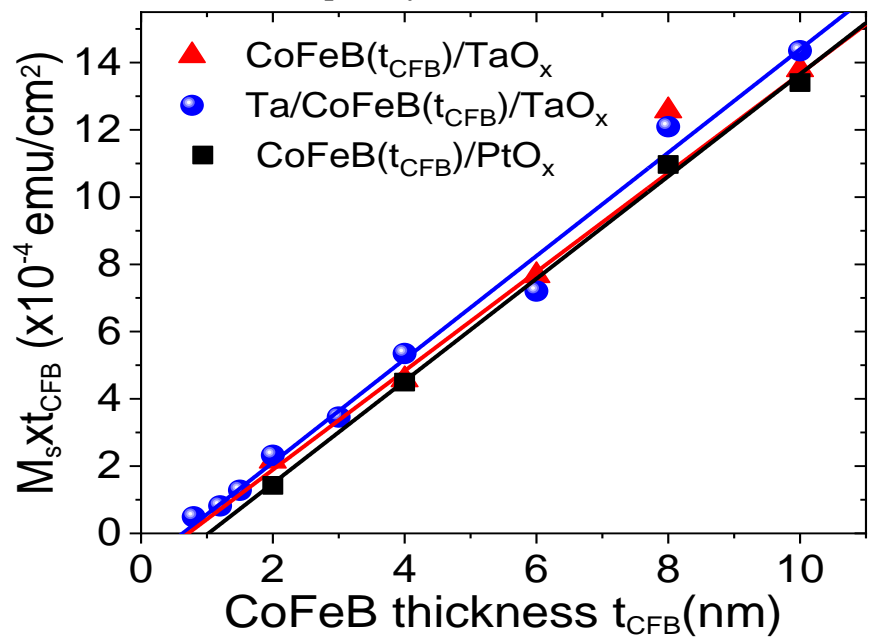

Fig. 1. Saturation magnetic moment per unit area versus thickness of the $\mathrm{CoFeB}$-based systems annealed at $225^{\circ} \mathrm{C}$. Symbols refer to experimental data and solid lines are linear fits.

For the gyromagnetic ratio $\gamma$ and consequently the g-factor, MS-FMR under perpendicular applied magnetic field has been used to measure the applied magnetic field dependence of the uniform precession mode frequency for unbuffered and Tabuffered $\mathrm{CoFeB} / \mathrm{TaO}_{\mathrm{x}}$ samples [29]. The obtained values of $\gamma / 2 \pi$ are $30.27 \pm 0.12 \mathrm{GHz} / \mathrm{T}(\mathrm{g}=2.16 \pm 0.1)$ and $28.86 \pm 0.14$ $\mathrm{GHz} / \mathrm{T}(\mathrm{g}=2.07 \pm 0.01)$, respectively. It is worth mentioning that the g-factor is known to be strongly influenced by surface and interface effects, as it depends on the local symmetry [30]. Therefore, it is not surprising to observe a buffer layer 
dependence of g-factor.

In the next paragraph, the focus is made on the investigation of effect of Ta buffer layer on PMA by measuring the effective magnetization $\left(4 \pi \mathrm{M}_{\mathrm{eff}}=4 \pi \mathrm{M}_{\mathrm{s}}-2 \mathrm{~K}_{\perp} / \mathrm{M}_{\mathrm{s}}\right)$ for each sample of $\mathrm{CoFeB} / \mathrm{TaO}_{\mathrm{x}}$-based systems. The perpendicular anisotropy constant $\left(\mathrm{K}_{\perp}\right)$ is then determined. To have $4 \pi \mathrm{M}_{\text {eff }}$, MS-FMR and BLS data, giving the in-plane applied magnetic field dependence of the uniform precession mode frequency (MSFMR) and mean frequency $\left(\mathrm{F}_{\mathrm{S}}+\mathrm{F}_{\mathrm{aS}}\right) / 2$, have been fitted using equation (2) reported in [29] and [31], respectively. Note that MS-FMR measurements revealed the existence of small uniaxial in-plane anisotropy, note exceeding 60 Oe. The linear dependence of $\mathrm{M}_{\mathrm{eff}}$ versus the inverse of the $\mathrm{CoFeB}$ effective thickness (shown in figure 2a) suggests the existence of surface magnetic anisotropy. $\mathrm{K}_{\perp}$ could therefore be phenomenologically separated in volume $\left(\mathrm{K}_{\mathrm{v}}\right)$ and interface $\left(\mathrm{K}_{\mathrm{s}}\right)$ contributions and approximately obeying the relation: $\mathrm{K}_{\perp}=\mathrm{K}_{\mathrm{v}}+\mathrm{K}_{\mathrm{s}} / \mathrm{t}_{\text {eff. }}$. The estimated interface anisotropy constants, deduced from the fit of MS-FMR (respectively BLS) data are $2.1 \pm 0.16 \mathrm{erg} / \mathrm{cm}^{2}\left(1.9 \pm 0.17 \mathrm{erg} / \mathrm{cm}^{2}\right)$ and $0.97 \pm 0.09 \mathrm{erg} / \mathrm{cm}^{2}$ for unbuffered and Ta-buffered $\mathrm{CoFeB} / \mathrm{TaO}_{\mathrm{x}}$ systems, respectively. The slight difference between the MS-FMR and BLS (about 10\%) is probably due to the difference in the applied magnetic field range when measuring the field dependence of the frequency, used to deduce $\mathrm{M}_{\mathrm{eff}}$ and to the more unknown parameters implied in the fit of the BLS data to

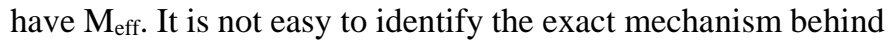
this difference in $K_{s}$ between Ta buffered and unbuffered $\mathrm{CoFeB} / \mathrm{TaO}_{\mathrm{x}}$ but one should precise the higher substrateinduced roughness. Indeed, atomic force microscopy measurements (not shown here) for $\mathrm{Si} / \mathrm{SiO}_{2}$ substrate and the as grown $\mathrm{Si} / \mathrm{SiO}_{2} / \mathrm{Ta}(3 \mathrm{~nm})$ have shown that the RMS roughness decreases after the growth of Ta. Such roughness creates inplane demagnetizing fields at edges of terraces reducing the shape anisotropy and therefore favors a higher interface PMA for $\mathrm{CoFeB}$ grown directly on $\mathrm{Si} / \mathrm{SiO}_{2}$.

It is worth mentioning the deviation of $\mathrm{M}_{\mathrm{eff}}$ from the linear dependence for thinner CoFeB films ( $\mathrm{t}_{\text {eff }}$ around $1.7 \mathrm{~nm}$ and 0.7 nm for unbuffered and Ta buffered systems) most probably due to the degradation of thinner $\mathrm{CoFeB}$ layers and the resulting lower Curie temperature. Therefore, although growing $\mathrm{CoFeB}$ directly on $\mathrm{Si} / \mathrm{SiO}_{2}$ increases drastically $\mathrm{K}_{\mathrm{s}}$, the sample quality degrades rapidly as $\mathrm{CoFeB}$ thickness decreases, making Ta buffer layer critical to develop spontaneous perpendicularly magnetized systems.

MS-FMR was used to measure the Gilbert damping $(\alpha)$ for $\mathrm{CoFeB} / \mathrm{TO}_{\mathrm{x}}$ and $\mathrm{Ta} / \mathrm{CoFeB} / \mathrm{TO}_{\mathrm{x}}$ by investigating the frequency dependence of the half width at half maximum height $(\Delta \mathrm{H})$. The magnetic field was applied in the film plane direction, giving the minimal $\Delta \mathrm{H}$ (determined from the variation of $\Delta \mathrm{H}$ versus the in-plane magnetic applied field direction). $\alpha$ was then deduced from the linear fit of the frequency dependence of $\Delta \mathrm{H}$. For both systems, $\alpha$ varies linearly with $1 / \mathrm{t}_{\mathrm{eff}}$ due the spin pumping, as shown in figure $2 \mathrm{~b}$. From the linear fit of these experimental data, it is then possible to determine values of CoFeB Gilbert damping $\alpha_{\mathrm{CFB}}$ and effective spin mixing conductance $g_{\text {eff }}^{\uparrow \downarrow}$ from the vertical axis intercept and the slope, respectively [29]. The obtained values of $\alpha_{\mathrm{CFB}}$ and $g_{e f f}^{\uparrow \downarrow}$ are $(2.1 \pm 0.4) \times 10^{-3},(3.1 \pm 0.19) \times 10^{-3},(16.4 \pm 2.1)$ and $(14.5 \pm 1.2) \mathrm{nm}^{-}$ ${ }^{2}$ for $\mathrm{CoFeB} / \mathrm{TaO}_{\mathrm{x}}$ and $\mathrm{Ta} / \mathrm{CoFeB} / \mathrm{TaO}_{\mathrm{x}}$, respectively. The lower (higher) value of $\alpha_{\mathrm{CFB}}\left(g_{\text {eff }}^{\uparrow \downarrow}\right)$ of $\mathrm{CoFeB} / \mathrm{TaO}_{\mathrm{x}}$ compared to that of $\mathrm{Ta} / \mathrm{CoFeB} / \mathrm{TaO}_{\mathrm{x}}$ are in line with the conclusion drawn above on the improvement of $\mathrm{K}_{\mathrm{s}}$ for the unbuffered $\mathrm{CoFeB} / \mathrm{TaO}_{\mathrm{x}}$. Moreover, the intermixing between the $\mathrm{CoFeB}$ and $\mathrm{Ta}$ at the lower interface could result in a relatively wide interface region which may kill the abrupt potential change, and conduction electrons across the $\mathrm{CoFeB} / \mathrm{Ta}$ interface are less scattered, resulting in small interface spin losses for $\mathrm{Ta} / \mathrm{CoFeB} / \mathrm{TaO}_{\mathrm{x}}$ system, as reported by $\mathrm{Ta}$ et al [32].
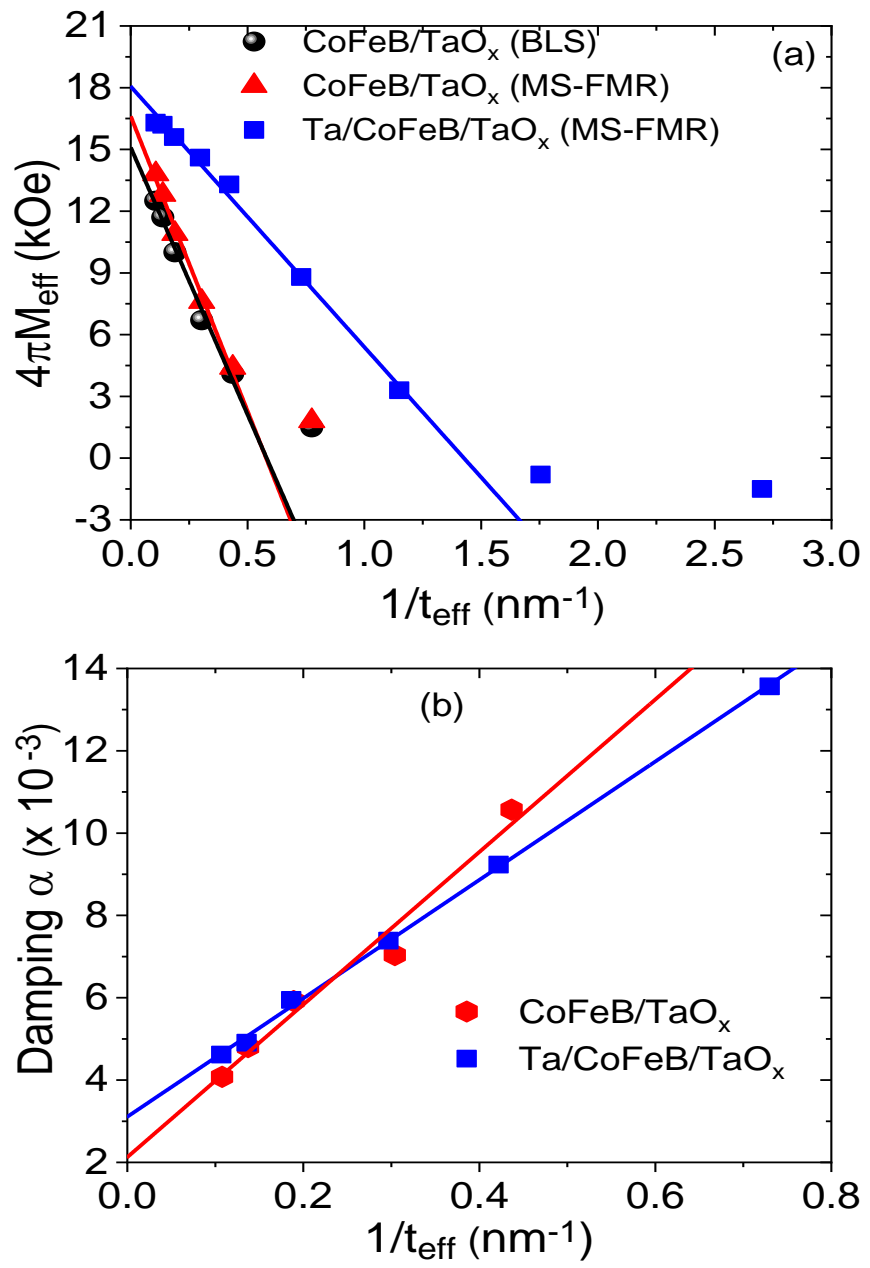

Fig. 2. (a) Effective magnetization ( $4 \pi \mathrm{M}_{\text {eff }}$ ) and (b) Gilbert damping constant as a function of the inverse effective thickness of $\mathrm{CoFeB}$ films for $\mathrm{CoFeB} / \mathrm{TO}_{x}$ and $\mathrm{Ta} / \mathrm{CoFeB} / \mathrm{TO}_{\mathrm{x}}$ systems. $4 \pi \mathrm{M}_{\text {eff }}$ values have been extracted from the fit of the in-plane applied magnetic field dependence of spin waves frequency measured by MS-FMR and BLS. Symbols refer to experimental data and solid lines are linear fits.

\section{B. 1- Effect of PtO $O_{x}$ thickness}

In the above investigation, the iDMI was not discussed due to its weak value in the $\mathrm{TaO}_{\mathrm{x}}$-based systems [29]. Therefore, the effect of oxidization on iDMI will be addressed for as grown and $225^{\circ} \mathrm{C}$ annealed $\mathrm{PtO}_{\mathrm{x}}$-based samples without Ta buffer layer owing to the strong iDMI generated by $\mathrm{Pt}$. The variation of the Pt thickness to be oxidized is powerful method to change 
the degree of oxidation at $\mathrm{CoFeB} / \mathrm{PtO}$ interface. Therefore, $\mathrm{CoFeB}(1.5 \mathrm{~nm}) / \mathrm{PtO}_{\mathrm{x}}\left(\mathrm{t}_{\mathrm{pt}}\right)$ grown directly on $\mathrm{Si} / \mathrm{SiO}_{2}$ substrates are considered in this study. For such ultrathin $\mathrm{CoFeB}$ films, BLS, in Damon-Eshbach configuration, has been used to investigate damping, PMA and iDMI. For iDMI, the thermally excited SW spectra have been recorded under in-plane saturating magnetic field, for each $\mathrm{ksw}_{\mathrm{sw}}$ within the range 4.1$20.45 \mu \mathrm{m}^{-1}$ and then fitted with a Lorentzian. The linear fit of the ksw dependence of the frequency mismatch $\Delta \mathrm{F}=\mathrm{F}_{\mathrm{S}}-\mathrm{F}_{\mathrm{aS}}$ was used to determine the iDMI strength, characterized by the effective constant $D_{\text {eff }}$ as given by equation (1).

$$
\Delta F=F_{S}-F_{a S}=D_{e f f} \frac{2 \gamma}{\pi M_{S}} k_{S W}=\frac{D_{S}}{t_{e f f}} \frac{2 \gamma}{\pi M_{S}} k_{S W}
$$

For PMA and damping, the recorded spectra at $\mathrm{k}_{\mathrm{sw}}=4.1 \mu \mathrm{m}$ ${ }^{1}$ and under variable in-plane applied magnetic fields were fitted with a Lorentzian to extract frequencies and the full width at half maximum linewidth $(\delta \mathrm{F})$ for $\mathrm{S}$ and aS lines. To cancel iDMI contribution, the mean values of frequencies and $\delta \mathrm{F}$ of $\mathrm{S}$ and aS lines were considered to deduce $\mathrm{K}_{\perp}$ and $\alpha$, respectively. Figure 3, shows the nominal Pt thickness (which is then oxidized) dependence of the obtained values of $\mathrm{K}_{\perp}, \alpha$ and $\mathrm{D}_{\text {eff }}$ using $\gamma / 2 \pi=30.27 \mathrm{GHz} / \mathrm{T}$ and $\mathrm{M}_{\mathrm{s}}=1520 \mathrm{emu} / \mathrm{cm}^{3}$. Note the similar behaviors of $\mathrm{K}_{\perp}, \alpha$ and $\mathrm{D}_{\text {eff }}$ for as deposited and annealed samples. $K_{\perp}$ and $\alpha$ (see figure $3 b$ ) decreases significantly with increasing $t_{P t}$. This is consistent with the increase of the CoFeB effective thickness. Indeed, when $t_{\mathrm{Pt}}$ increases $\mathrm{CoFeB}$ is less oxidized, $t_{d}$ is reduced and consequently $t_{\text {eff }}$ is enhanced as confirmed by the increasing of the areal magnetic moment shown in the inset of figure 3a. It is well known that $D_{\text {eff }}$ varies linearly with $1 / t_{\text {eff }}$ for sufficient $t_{\text {eff }}$. However, when the FM thickness is lower than a critical value, $D_{\text {eff }}$ decreases with $1 / t_{\text {eff }}$ since iDMI requires a finite thickness for its full emergence and thus its magnitude is maximized [33]. Therefore, owing to the thick $\mathrm{t}_{\mathrm{d}}$ in $\mathrm{CoFeB} / \mathrm{PtO}_{\mathrm{x}}$ systems and the $1.5 \mathrm{~nm}$ nominal thickness of $\mathrm{CoFeB}$, $\mathrm{t}_{\mathrm{eff}}$ should be largely below $1 \mathrm{~nm}$ and thus $\mathrm{D}_{\text {eff }}$ should increase with $\mathrm{t}_{\mathrm{Pt}}$ as explained above. To validate this hypothesis, the $t_{\text {eff }} \times M_{s}$ deduced from the VSM measurements (inset figure 3a) have been used in equation 1 to evaluate $\mathrm{D}_{\mathrm{s}}$. as shown in the inset of figure $3 \mathrm{c}$. The variation of $D_{\mathrm{s}}$ shows that $\mathrm{t}_{\mathrm{eff}}$ increases but remains lower than the critical value above which $\mathrm{D}_{\mathrm{s}}$ should be constant. Conversely $g_{\text {eff }}^{\uparrow \downarrow}$, deduced from damping values using $\alpha_{\mathrm{CFB}}=2.1 \times 10^{-3}, \mathrm{t}_{\mathrm{eff}} \times \mathrm{M}_{\mathrm{s}}$ and equation (4) reported in [30], is nearly constant as expected, confirming the peculiar behavior of $\mathrm{D}_{\text {eff }}$ below the iDMI full emergence thickness.

Note that for annealed samples with $\mathrm{PtO}_{\mathrm{x}}$ thicknesses below $1 \mathrm{~nm}$, no signal has been detected, suggesting that such samples are not magnetic at room temperature, likely because of an increased oxidation of $\mathrm{CoFeB}$ layer after annealing. Furthermore, annealing increases $K_{\perp}$ and $\alpha$ while $t_{d}$ and $D_{\text {eff }}$ are only slightly affected suggesting an enhancement of the $\mathrm{CoFeB}$ crystallinity and interfaces.

It is worth mentioning that linear correlation between $\mathrm{K}_{\perp}$ and $\alpha$ (not shown here) was obtained for both $\mathrm{Ta} / \mathrm{CoFeB}(\mathrm{tCoFeB}) / \mathrm{TaOx}, \quad \mathrm{CoFeB}(\mathrm{tCoFeB}) / \mathrm{TaOx}$ and as grown $\mathrm{CoFeB}(\mathrm{tCoFeB}) / \mathrm{PtOx}$ due to the second order the dependence of the two parameters with SOC as expected theoretically.
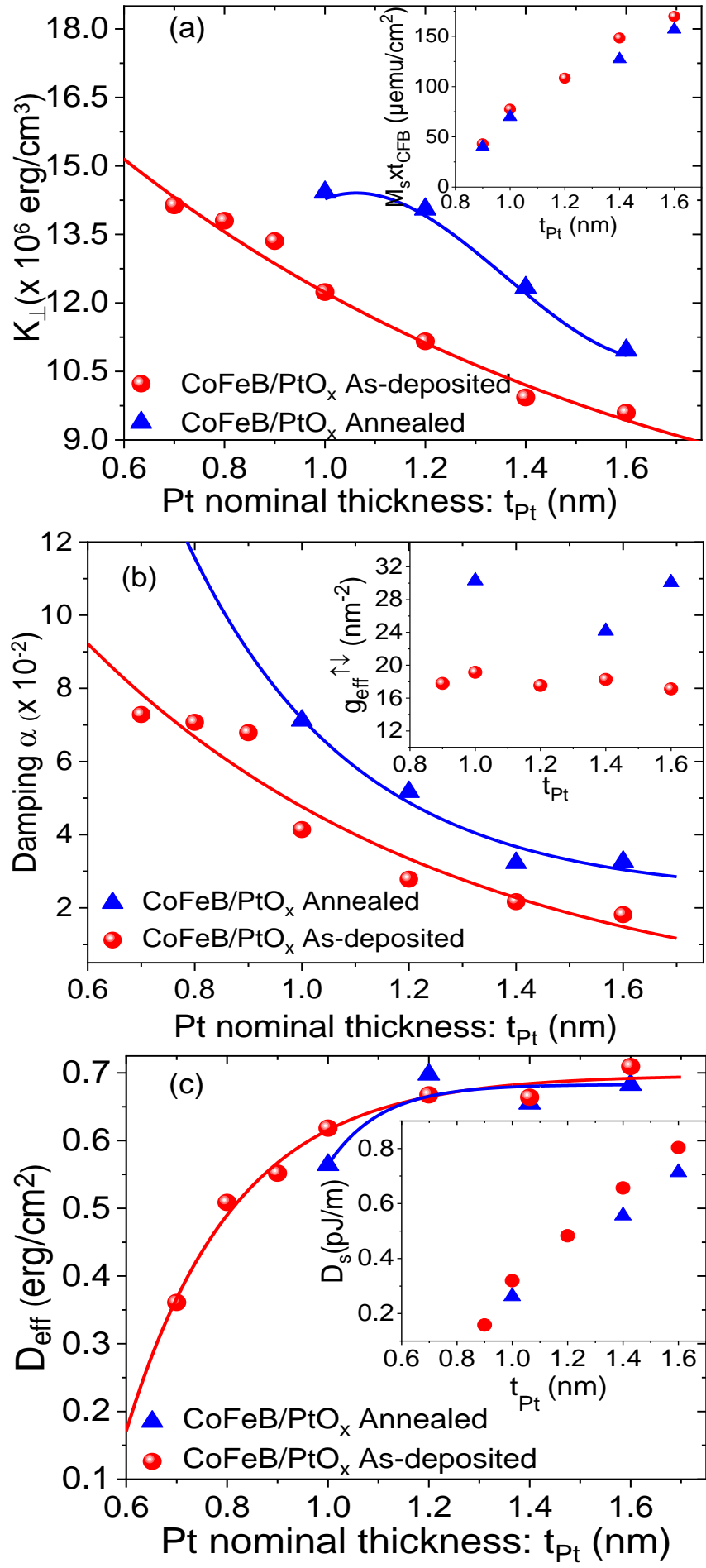

Fig. 3. Variations of (a) perpendicular anisotropy constant $\left(\mathrm{K}_{\perp}\right)$, (b) Gilbert damping and (c) effective iDMI constant $\left(\mathrm{D}_{\text {eff }}\right)$ as a function of the nominal $\mathrm{Pt}$ thickness $\left(\mathrm{t}_{\mathrm{Pt}}\right)$ for the as grown and the $225{ }^{\circ} \mathrm{C}$ annealed $\mathrm{CoFeB}(1.5 \mathrm{~nm}) / \mathrm{PtO}_{\mathrm{x}}$ films. $\mathrm{PtO}_{\mathrm{x}}$ is obtained by oxidization of $\mathrm{Pt} \mathrm{t}_{\mathrm{Pt}}$ indicated in the horizontal axis. Symbols refer to experimental data and solid lines are eye guides. Insets shows (a) areal magnetic moment, (b) effective spin mixing conductance and (c) surface iDMI constant versus $t_{\mathrm{Pt}}$.

\section{CONCLUSION}

We demonstrated that the underlayer nature and the capping layer thickness play a decisive role in tuning PMA, iDMI and 
spin pumping-induced damping, confirming their interfacial origin and their spin-orbit coupling correlation. Indeed, large surface PMA was found in the unbuffered $\mathrm{CoFeB} / \mathrm{TaO}_{\mathrm{x}}$ system and degraded for ultra-thin $\mathrm{CoFeB}$ films, making Ta buffer layer critical to develop spontaneous perpendicularly magnetized systems probably due to the different substrate induced roughness. The effect of $\mathrm{PtO}_{\mathrm{x}}$ thickness on PMA, iDMI and spin pumping induced damping was investigated in asdeposited and $225^{\circ} \mathrm{C}$ annealed $\mathrm{CoFeB} / \mathrm{PtO}_{\mathrm{x}}$ systems: a strong dependence of these three parameters with $\mathrm{PtO}_{\mathrm{x}}$ thickness was observed. This was mainly attributed to variation of the dead layer, giving another opportunity to control these parameters depending on the desired application.

\section{ACKNOWLEDGMENT}

This research was supported by 'structure fédérative de recherche NAP MOSAIC' of the University Sorbonne Paris Nord, Conseil regional d'île-de-France (convention 1763) through the DIM NanoK (BIDUL project) and the French ANR (contract ELECSPIN n ANR-16-CE24-0018, contract ADMIS $\mathrm{n}^{\circ}$ ANR-19-CE24-0019), and DARPA TEE program through Grant No. MIPR HR0011831554.

\section{REFERENCES}

[1] M. N. Baibich, J. M. Broto, A. Fert, F. Nguyen Van Dau, F. Petro, P. Etienne,G. Creuzet, A. Friederich, and J. Chazelas, "Giant Magnetoresistance of (001) Fe/ (001) Cr Magnetic Superlattices," Phys. Rev. Lett., vol. 61, (21), pp. 2472-2475, 1988.

[2] G. Binasch, P. Grünberg, F. Saurenbach, and W. Zinn, "Enhanced magnetoresistance in layered magnetic structures with antiferromagnetic interlayer exchange," Physical Review B, vol. 39(7), pp. 4828-4830, 1989.

[3] B. Tudu and A. Tiwari, "Recent Developments in Perpendicular Magnetic Anisotropy Thin Films for Data Storage Applications," Vacuum, vol. 146, pp. 329-341, 2017.

[4] J.G. Zhu and C. Park, "Magnetic tunnel junctions," Materialstoday, vol. 9(11), pp. 36-45, 2006.

[5] A. Fert, N. Reyren and V. Cros, "Magnetic skyrmions: advances in physics and potential applications," Nat. Mat., vol. 2, pp. 17031, 2017.

[6] X. Zhang, Y. Zhou, K. Mee Song, T-E. Park, J. Xia, M. Ezawa, X. Liu, W. Zhao, G. Zhao and S. Woo, "Skyrmion-electronics: writing, deleting, reading and processing magnetic skyrmions toward spintronic applications," J. Phys.: Condens. Matter, vol. 32, pp. 143001, 2020.

[7] M. T. Johnson, P. J. H. Bloemenz, F. J. A. den Broeder, and J. J. de Vries, "Magnetic anisotropy in metallic multilayers," Rep. Prog. Phys., vol. 59, pp. 1409, 1996.

[8] A. Brataas, Y. Tserkovnyak, G. E.W. Bauer and B. I Halperin, "Spin battery operated by ferromagnetic resonance," Phys. Rev. B, vol. 66, pp. 060404, 2002.

[9] Y. Tserkovnyak, A. Brataas, G. E.W. Bauer and B. I Halperin, "Nonlocal magnetization dynamics in ferromagnetic heterostructures", Rev. Mod. Phys., vol. 77, pp. 1375, 2005.

[10] I. Dzyaloshinsky, "A thermodynamic theory of weak ferromagnetism of antiferromagnetics," J Phys. Chem. Sol, vol. 4, pp. 241-255, 1958.

[11] T. Moriya, "New Mechanism of Anisotropic Superexchange Interaction," Phys. Rev. Lett., vol. 4, pp. 228, 1960.

[12] S. Bhatti, R. Sbiaa, A. Hirohata, H. Ohno, S. Fukami, and S.N. Piramanayagam, "Spintronics based random access memory: a review," Materials today, vol. 20, pp. 530-548, 2017.

[13] B. Rodmacq, S. Auffret, B. Dieny, S. Monso, and P. Boyer, "Crossovers from in-plane to perpendicular anisotropy in magnetic tunnel junctions as a function of the barrier degree of oxidation," J. Appl. Phys., vol. 93, pp. $7513,2003$.

[14] B. Dieny and M. Chshiev, "Perpendicular magnetic anisotropy at transition metal/oxide Interfaces and applications," Rev. Mod. Phys., vol. 89, pp. 025008, 2017.
[15] S. Ikeda, J. Hayakawa, Y. Min Lee, F. Matsukura, Y. Ohno, T. Hanyu, and H. Ohno, "Magnetic tunnel junctions for spintronic memories and beyond," IEEE Trans. Elec. Dev., vol. 54 (5), pp. 991-1002, 2007.

[16] S. Peng, D. Zhu, J. Zhou, B. Zhang, A. Cao, M. Wang, W. Cai, K. Cao, and W. Zhao, "Modulation of heavy metal/ferromagnetic metal Interface for high-performance spintronic devices,". Adv. Electron. Mater., vol. 5, pp.1900134, 2019.

[17] D. M. Lattery, D. Zhang, J. Zhu, X. Hang, J-P. Wang and X. Wang, "Low Gilbert Damping Constant in Perpendicularly Magnetized W/CoFeB/MgO Films with High Thermal Stability," Sc. Rep., vol. 8, pp. 13395, 2018.

[18] M. Uchida, Y. Onose, Y. Matsui, and Y. Tokura, "Real-Space Observation of Helical Spin Order," Science, vol. 311, pp.359-361 2006.

[19] M. Bode, M. Heide, K. von Bergmann, P. Ferriani, S.Heinze, G. Bihlmayer, A. Kubetzka, O. Pietzsch, S. Blügel, and R. Wiesendanger, "Chiral magnetic order at surfaces driven by inversion asymmetry ," Nature, vol. 447, pp. 190, 2007.

[20] A. Tonomura, X. Yu, K. Yanagisawa, T. Matsuda, Y. Onose, N. Kanazawa, H. S. Park and Y. Tokura, "Real-Space Observation of Skyrmion Lattice in Helimagnet MnSi Thin Samples," Nano Lett., vol. 12, pp. 1673-1677, 2012.

[21] A. Fert, V. Cros and J. Sampaio, "Skyrmions on the track," Nat. nanotech., vol. 8, pp. 152-156, 2013.

[22] A. Thiaville, S. Rohart, E. Jué, V. Cros, and A. Fert, "Dynamics of Dzyaloshinskii domain walls in ultrathin magnetic films," $E P L$, vol. 100, pp. 57002, 2012.

[23] H. X. Yang, O. Boulle, V. Cros, A. Fert, and M. Chshiev, “Controlling Dzyaloshinskii-Moriya Interaction via Chirality Dependent AtomicLayer Stacking, Insulator Capping and Electric Field," Sci. Rep., vol. 8, pp. 12356, 2018.

[24] A. Belabbes, G. Bihlmayer, S. Blügel, and A. Manchon, "Oxygen-enabled control of Dzyaloshinskii-Moriya Interaction in ultra-thin magnetic films," Sci. Rep., vol.6, pp. 24634, 2016.

[25] H. T. Nembach, E. Jué, E. R. Evarts and J. M. Shaw, "Correlation between Dzyaloshinskii-Moriya interaction and orbital angular momentum at an oxide-ferromagnet interface," Phys. Rev. B, vol. 101, pp. 020409(R), 2020.

[26] M. Arora, J. M. Shaw and H. T. Nembach, "Variation of sign and magnitude of the Dzyaloshinskii-Moriya interaction of a ferromagnet with an oxide interface," Phys. Rev. B, vol. 101, pp. 054421, 2020.

[27] T. Srivastava, M. Schott, R. Juge, V. Křižáková, M. Belmeguenai, Y. Roussigné, A. Bernand-Mantel, L.t Ranno, S. Pizzini, S-M. Chérif, A. Stashkevich, S. Auffret, O. Boulle,G. Gaudin, M. Chshiev, C. Baraduc, H. Béa, "Large-Voltage Tuning of Dzyaloshinskii-Moriya Interactions: A Route toward Dynamic Control of Skyrmion Chirality," Nano Lett. vol., 18, pp. 4871-4877, 2018.

[28] T. Srivastava, W. Lim, I. Joumard, S. Auffret, C. Baraduc and H. Béa, "Mapping different skyrmion phases in double wedges of Ta/FeCoB/TaOx trilayers," Phys. Rev. B, vol. 100, pp. 220401(R), 2019.

[29] I. Benguettat-El Mokhtari, Y. Roussigné, S. M. Chérif, A. Stashkevich, S. Auffret, C. Baraduc, M. Gabor, H. Béa, and M. Belmeguenai, "Interface phenomena in ferromagnet/TaOx-based systems: Damping, perpendicular magnetic anisotropy, and Dzyaloshinskii-Moriya interaction," Phys. Rev. Mat., vol. 4, pp. 124408, 2020.

[30] C. Le Graët, D. Spenato, N. Beaulieu, D. Dekadjevi, J-Ph. Jay, S. Pogossian, B. Warot-Fonrose, J. Ben Youssef, "Driving mechanism for damping and g-factor innon-amorphous ferromagnetic CoFeZr ultrathin films," EuroPhys. Lett., vol. 115, pp. 17002, 2016.

[31] I. Benguettat-El Mokhtari, D. Ourdani, Y. Roussigné, R. B. Mos, M. Nasui, F. Kail, L. Chahed, S. M. Chérif, A. Stashkevich, M. Gabor, nd M Belmeguenai, "Perpendicular magnetic anisotropy and interfacial Dzyaloshinskii-Moriya interaction in as grown and annealed $\mathrm{X} / \mathrm{Co} / \mathrm{Y}$ ultrathin systems," Phys.: Condens. Matter, vol. 132, pp. 495802, 2020.

[32] X. Tao, Q. Liu, B. Miao, R. Yu, Z. Feng, L. Sun, B. You, J. Du, K. Chen, S. Zhang, L. Zhang, Z. Yuan, D. Wu, H. Ding, Sci. Adv. Vol. 4, pp.eaat1670, 2018.

[33] Y-K. Park, J-S. Kim, Y-S. Nam, S. Jeon, J-H. Park, K-W. Kim, H-W. Lee, B-C. Min, and S-B. Choe, "Interfacial atomic layers for full emergence of interfacial Dzyaloshinskii-Moriya interaction," NPG Asia Materials, vol. 12, pp. 38, 2020. 